\title{
Some Ideas on Calculating Great Circle Sailings
}

\author{
Chung-hsing Chiang and An-yuang Tseng \\ (National Taiwan Ocean University)
}

1. INTRODUCTION. To calculate the position of the vertex of a great circle between two given positions, it has been usual to use the cosine formula followed by Napier's rules. One expects first to calculate the distance and the initial course before proceeding to find the vertex. For a particular departure position, there is an infinite number of great circles and vertices. The relationship between the latitude and longitude of such a family of vertices has been investigated and this has resulted in the identification of what has tentatively been termed a 'vertex circle'. This has led to a simpler method of calculation which is presented for discussion.

2. METHODOLOGY. In the general case, the two vertices and the two points where a great circle cuts the equator partition the great circle into four equal parts, each being $90^{\circ}$ in length. We consider a fixed departure point (A in Fig. 1 ) and a movable point $\mathrm{E}$ where a great circle cuts the equator on the opposite side of $A$ from the nearest vertex $\mathrm{V}$. We now imagine the arc EV as a stick of fixed length $90^{\circ}$, such that it always passes through point $A$. As the end $E$ moves along the equator towards the meridian $(M)$ through $A$, the latitude of $V$ at the other end of the stick increases as shown in Fig. $I$. When $E$ reaches $M$, the vertex coincides with the pole $(P)$. As $E$ continues past $M$, the latitude of $V$ decreases until it finally arrives back at $A$. The path of $V$ as point $E$ moves along the equator thus forms a closed curve, which is tentatively termed the 'vertex circle' for position A.

Considerations of symmetry imply that the vertex remote from A traces out a similar curve in the opposite hemisphere.

3. DEVELOPMENT. In section 2, the concept of a vertex circle relating to a particular departure position A was discussed. Clearly, there will be a similar vertex circle for any given destination position B (see Figure 2). The two vertex circles intersect at the pole $(\mathrm{P})$, but they also intersect at a second point which is the vertex of the great circle which passes through $\mathrm{A}$ and $\mathrm{B}$.

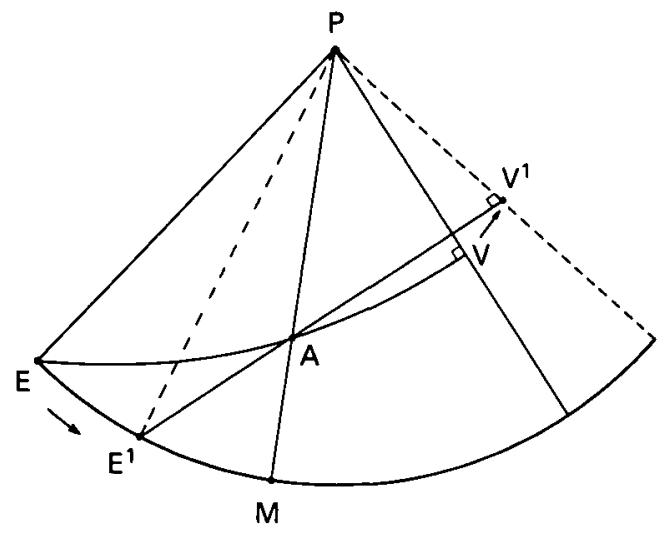

Fig. I. Positions of vertices for a family of great circles through A 


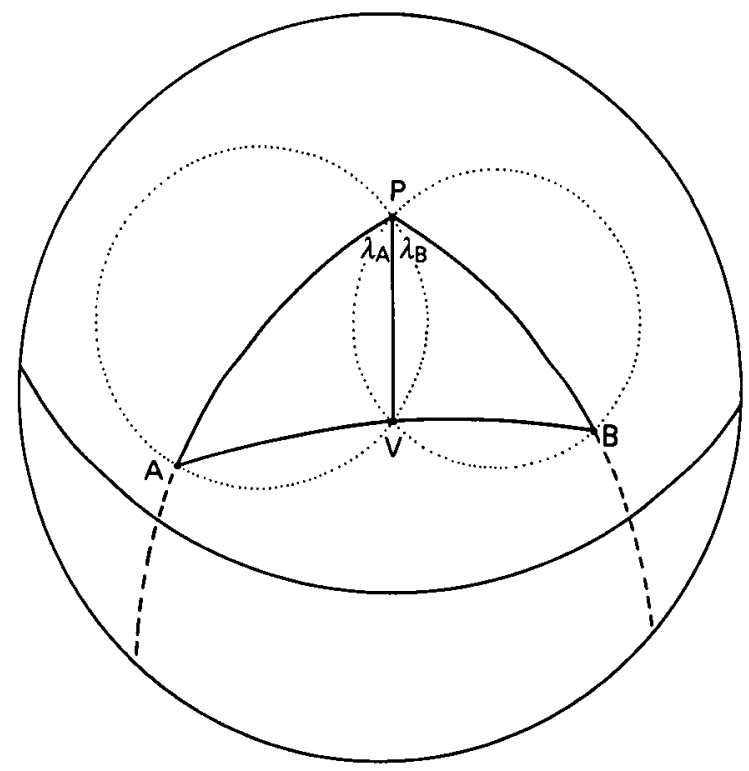

Fig. 2. Intersecting vertex circles for positions A and B

Referring to Fig. 2, and using Napier's rules, in triangle PAV, we have:

$$
\cos \lambda_{\mathrm{A}}=\tan \phi_{\mathrm{A}} \cot \phi_{\mathrm{V}},
$$

where $\lambda_{A}$ is the difference in longitude between $A$ and $V, \phi_{A}$ is the latitude of $A$ and $\phi_{\mathrm{V}}$ is the latitude of $\mathrm{V}$. We also note that:

$$
\phi_{\mathrm{A}} \leqslant \phi_{\mathrm{V}} \leqslant 90^{\circ} \text { and } 0 \leqslant \mathrm{P} \leqslant 90^{\circ}
$$

Similarly, in relation to the destination, in triangle PBV, we have:

$$
\cos \lambda_{\mathrm{B}}=\tan \phi_{\mathrm{B}} \cot \phi_{\mathrm{V}},
$$

where $\lambda_{B}$ is the difference of longitude between the vertex and $B$, and $\phi_{B}$ is the latitude of B. Dividing 2 by 1 gives :

$$
\begin{gathered}
\frac{\cos \lambda_{\mathrm{B}}}{\cos \lambda_{\mathrm{A}}}=\frac{\tan \phi_{\mathrm{B}}}{\tan \phi_{\mathrm{A}}}, \\
\frac{\cos \left(\lambda-\lambda_{\mathrm{A}}\right)}{\cos \lambda_{\mathrm{A}}}=\frac{\tan \phi_{\mathrm{B}}}{\tan \phi_{\mathrm{A}}}
\end{gathered}
$$

where $\lambda$ is the difference of longitude between $A$ and $B$,

$$
\begin{gathered}
\cos \lambda+\sin \lambda \tan \lambda_{\mathrm{A}}=\tan \phi_{\mathrm{B}} \cot \phi_{\mathrm{A}} \\
\tan \lambda_{\mathrm{A}}=\tan \phi_{\mathrm{B}} \cot \phi_{\mathrm{A}} \operatorname{cosec} \lambda-\cot \lambda .
\end{gathered}
$$

4. APPLICATION. In order to find the position of the vertex of a great circle between given positions $A$ and $B$, the difference of longitude $\left(\lambda_{A}\right)$ between $A$ and the vertex is first calculated using equation (3).

Knowing $\lambda_{\mathrm{A}}$ and $\phi_{\mathrm{A}}$ in right-angled triangle $\mathrm{PAV}$, the remaining parts may be calculated using Napier's rules. In particular, the latitude of the vertex $\left(\phi_{\mathrm{V}}\right)$, the initial 
course (angle $A$ ) and the distance to the vertex (AV). It is straightforward thence to solve right-angled triangle PVB for the distance (VB) and, if required, the final course (angle B).

The great circle course at chosen intermediate points, such as $\mathrm{X}$, along the great circle track can be calculated in the usual way by applying Napier's rules to triangles such as PVX.

5. CONCLUSIONS. The method discussed in this paper provides a simpler and more direct way of calculating great circle track parameters than the usual practice, which requires the application of the awkward cosine formula twice. The usual procedure (see, e.g. Cotter $1953^{1}$ ) uses the cosine formula to find first of all the great circle distance and then the initial course, before the simpler Napier rules can be used to find the position of the vertex.

It remains to be determined whether the title 'vertex circle' is appropriate and what is the nature of the curve on the surface of the Earth. Also, whether the concept has further applications.

\title{
REFERENCES
}

' Cotter, C. H. (1953). The Elements of Navigation. Sir Isaac Pitman \& Sons, London.

\author{
KEY WORDS
}

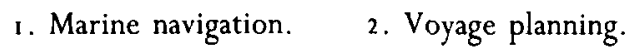

\section{The 'Two-Body Problem' At Sea}

\section{Mike Pepperday}

Navigation by direct computation of position from observations to two bodies was used by the author at sea. Calculation of the incorrect of the possible two solutions was avoided, but the method was still found inconvenient because it copes only with two bodies; it cannot cope with more than one sight to each body, and is of no use when there is only one body. The standard intercept method copes with all these situations and is mathematically more elegant.

Many people have pointed out that, if the altitudes of two astronomical bodics are measured, the observer's position can theoretically be directly calculated. How does the approach work out in practice on the high seas?

Chicsa and Chicsa' give some mathematics to solve for position, mentioning that they have published a manual with a BASIC program. Following from them Spencer, ${ }^{2}$ describes an approach using an $8 \mathrm{~K}$ Sharp $\mathrm{PC}_{1} 500$ pocket computer. Bowditch ${ }^{3}$ set out a proposal made by one Charles T. Dozier in 1949 , Bennett ${ }^{4}$ set out a general solution with a worked example and the Appendix here gives a short solution for a calculator.

The 'two-body problem' seems to surface periodically. Bennett quoted Sadler ${ }^{5}$ as remarking in 1977 that it has been discussed and investigated often. I think what draws us to a two-body solution is a perception that the Marcq St Hilaire or intercept method is mathematically inelegant. Why use an estimate of your position if you can solve directly for your true position? Spencer says it 'seems rather incongruous ' and expresses the hope that small boats will use 'this convenient method'. Chiesa and Chiesa say, 